\title{
A de novo nonsense PDGFB mutation causing idiopathic basal ganglia calcification with laryngeal dystonia
}

\author{
Gaël Nicolas ${ }^{1,2,3,4}$, Agnès Jacquin ${ }^{5}$, Christel Thauvin-Robinet ${ }^{6}$, Anne Rovelet-Lecrux ${ }^{1,2,4}$, Olivier Rouaud ${ }^{5}$, \\ Cyril Pottier ${ }^{1,2,4}$, Marie-Hélène Aubriot-Lorton ${ }^{7}$, Stéphane Rousseau ${ }^{1,2,4}$, David Wallon ${ }^{1,2,4,8}$, \\ Christian Duvillard $^{9}$, Yannick Béjot ${ }^{5}$, Thierry Frébourg ${ }^{1,2,3}$, Maurice Giroud ${ }^{5}$, Dominique Campion ${ }^{1,2,4,10,11}$ \\ and Didier Hannequin ${ }^{\star, 1,2,3,4,8,11}$
}

Idiopathic basal ganglia calcification (IBGC) is characterized by brain calcification and a wide variety of neurologic and psychiatric symptoms. In families with autosomal dominant inheritance, three causative genes have been identified: SLC20A2, PDGFRB, and, very recently, PDGFB. Whereas in clinical practice sporadic presentation of IBGC is frequent, well-documented reports of true sporadic occurrence are rare. We report the case of a 20 -year-old woman who presented laryngeal dystonia revealing IBGC. Her healthy parents' CT scans were both normal. We identified in the proband a new nonsense mutation in exon 4 of PDGFB, c.439C $>$ T (p.GIn147*), which was absent from the parents' DNA. This mutation may result in a loss-of-function of PDGF-B, which has been shown to cause IBGC in humans and to disrupt the blood-brain barrier in mice, resulting in brain calcification. The c.439C $>T$ mutation is located between two previously reported nonsense mutations, c.433C $>T$ (p.Gln $145^{*}$ ) and c.445C $>\mathrm{T}$ (p.Arg149*), on a region that could be a hot spot for de novo mutations. We present the first full demonstration of the de novo occurrence of an IBGC-causative mutation in a sporadic case.

European Journal of Human Genetics (2014) 22, 1236-1238; doi:10.1038/ejhg.2014.9; published online 12 February 2014

Keywords: IBGC; Fahr's disease; PDGFB; PDGFRB; de novo; dementia

\section{INTRODUCTION}

Idiopathic basal ganglia calcification (IBGC) is defined by the presence of brain calcification affecting at least the basal ganglia, after the exclusion of known causes. There is wide intra- and interfamilial diversity of symptoms and ages of onset. The most common signs are cognitive impairment, psychiatric signs, movement disorders, gait disorder, dysarthria, cerebellar syndrome, pyramidal signs, and seizures. ${ }^{1}$ Most familial cases are dominantly inherited. Three causative genes have recently been discovered. First, loss-of-function mutations in SLC20A2, causing IBGC in approximately $40 \%$ families, 2,3 might have an impact on the inorganic phosphate metabolism in the brain. Then, we recently identified PDGFRB, which encodes the transmembrane receptor platelet derived growth factor receptor $\beta$ (PDGFR $\beta)$, as a second IBGC causative gene. ${ }^{4}$ Third, loss of function of PDGF-B (encoded by the PDGFB gene), the main ligand of $\operatorname{PDGFR} \beta$, has recently been shown to cause IBGC in humans and brain calcification through a disruption of the bloodbrain barrier integrity in mice. ${ }^{5}$ Sporadic presentation of IBGC is not uncommon. Nevertheless, true sporadic cases, defined by the absence of basal ganglia calcification in the parents' CT scans after the age of 50, ${ }^{6}$ were rarely reported. ${ }^{7}$ We report the case of a 20 -year-old woman presenting laryngeal dystonia revealing IBGC, due to a de novo nonsense mutation within PDGFB.

\section{MATERIALS AND METHODS}

The proband and her parents gave informed, written consent. The study was approved by our local ethics committee. Genomic DNA was extracted from whole peripheral blood using the Flexigen DNA kit (Qiagen, Hilden, Germany). In the proband's DNA, the entire coding sequence and intron/ exon boundaries of SLC20A2 (NM_006749.3), PDGFRB (NM_002609.3), and PDGFB (NM_002608.2) were PCR-amplified. PCR products were sequenced using the BigDye V3.1 Terminator Kit (Applied Biosystems, Courtaboeuf, France) on an automated sequencer (ABI 3100; Applied Biosystems). After the identification of the nonsense mutation in exon 4 of PDGFB (the exons were numbered according to the reference NM_002608.2 in this report; exon 4 corresponds to exon 5 using NG_012111.1 as reference) in the proband, we performed PCR amplification and sequencing of this exon in the parents.

To screen the presence of the mutation in controls, we used the 6503 exomes provided by the Exome Variant Server (EVS, NHLBI GO exome sequencing project, accessed in August, 2013), and a series of 173 in-house exomes from individuals with no neuropsychiatric disorder. To ascertain the parenthood, we used four informative microsatellites (D1S439, D9S1784, D14S986, and D19S913) and genotyped the proband and her parents using a multiplex fluorescent PCR.

${ }^{1}$ Inserm U1079, Rouen, France; ${ }^{2}$ IRIB, Normandie University, Rouen, France; ${ }^{3}$ Department of Genetics, Rouen University Hospital, Rouen, France; ${ }^{4}$ CNR-MAJ, Lille, Paris-Salpêtrière and Rouen University Hospitals, Paris, France; ${ }^{5}$ Department of Neurology, Dijon University Hospital, Dijon, France; ${ }^{6}$ Centre de Génétique et Centre de Référence Anomalies du Développement et Syndromes Malformatifs de l'interrégion Grand-Est, Hôpital d'Enfants, Dijon University Hospital, Dijon, France; ${ }^{7}$ Department of Pathology, Dijon University Hospital, Dijon, France; ${ }^{8}$ Department of Neurology, Rouen University Hospital, Rouen, France; ${ }^{9}$ Department of otolaryngology and head and neck surgery, Dijon University Hospital, Dijon, France; ${ }^{10}$ Department of Research, Rouvray Psychiatric Hospital, Sotteville-lès-Rouen, France

*Correspondence: Professor D Hannequin, service de Neurologie, CHU de Rouen, 1, rue de Germont, Rouen cedex 76031 , France. Tel: +33 232 888740 ; Fax: +33 232888 7417; E-mail: didier.hannequin@chu-rouen.fr

${ }^{11}$ These authors contributed equally to this work

Received 29 August 2013; revised 22 November 2013; accepted 7 January 2014; published online 12 February 2014 
To search for nonsense-mediated RNA decay (NMD), we reverse transcribed total mRNA collected in PAXgene Blood RNA tubes (Qiagen) from the patient and her parents (Fisher Scientific, Illkirch, France) and performed PCR sequencing of the complementary DNA of Exon 4 of PDGFB. We then designed a reverse transcription-quantitative multiplex PCR of short fluorescent fragments (RT-QMPSF) of two amplicons of PDGFB, with TOP1 and SF3A1 genes as controls.

All primer sequences are reported in Supplementary Material. The mutation was submitted to the Leiden Open Variation Database (LOVD).

\section{RESULTS}

\section{Case report}

A 20-year-old woman was hospitalized for dysphagia and stridor. She had no familial medical history, and her personal medical history was marked by migraine without aura, leading to the identification of faint calcification of both lenticular nuclei in a CT scan performed at 10 years old during a migraine episode. Direct laryngoscopy showed bilateral vocal cord dysfunction: when the patient breathed in, the vocal cords moved in adduction. Three days later, she presented an acute exacerbation of dyspnea, with vocal cord paralysis in adduction, leading to oro-tracheal intubation. As it was not possible to deprive the patient from the ventilator, she underwent a tracheotomy. Neurological examination, laryngeal CT scan, and electromyography were all normal. No loco-regional cause was found. The cerebral CT scan showed calcification of both lenticular and caudate nuclei, and FLAIR-weighted magnetic resonance imaging only showed several punctiform hyperintensities in the supratentorial white matter and discrete anterior periventricular hyperintensities (Figure 1). An extensive etiological assessment of brain calcification (according to Nicolas $e a^{4}$ ) was negative. We retained the diagnosis of IBGC. Her healthy parents had normal CT-scans, performed at the age of 52 years (mother) and 66 years (father). Muscle biopsy (performed as a search for mitochondrial disease) did not show any calcification.

\section{Genetics}

No potentially pathogenic variant was identified in SLC20A2 and $P D G F R B$ in the proband. We found a heterozygous single-nucleotide substitution in exon 4 of $P D G F B, c .439 \mathrm{C}>\mathrm{T}$, predicted to introduce a premature stop codon at position 147 in the protein (p.Gln147*). This mutation was not retrieved in the parents and was absent in controls. Microsatellite analysis was compatible with paternity. We retrieved the mutation at the RNA level, r.439c > u (NM_002608.2). We did not find any decrease in the expression of the mutated allele in the proband by RT-PCR sequencing and RT-QMPSF (Supplementary Data). However, as no SNP was present in the $P D G F B$-coding sequence, we could not use a more sensitive approach, such as an SNaPshot technique, to detect NMD.

In addition, with the aim to find more mutations of $P D G F B$, we also sequenced the entire coding region and exon-intron boundaries of PDGFB in the 12 index cases from IBGC families and the 17 cases with sporadic presentation of IBGC with no mutation within PDGFRB and SLC20A2 from our French case series. ${ }^{1}$ We only found two mutations: the first one was already reported within the original article, ${ }^{5}$ and the second one is the currently presented mutation.

\section{DISCUSSION}

Our patient presented laryngeal dystonia, which is defined by involuntary contraction of the vocal cords, responsible for dysphonia. ${ }^{8}$ Severe laryngeal dystonia may also be responsible for life-threatening dyspnea. Laryngeal dystonia is a rare manifestation of IBGC $^{9}$ and such a severe and acute revelation of IBGC has never been reported, to our knowledge.

IBGC is usually inherited according to an autosomal dominant pattern. As clinical expression of brain calcification is not constant, clinically sporadic presentation of IBGC may be due to mutations inherited from an asymptomatic parent in some cases.

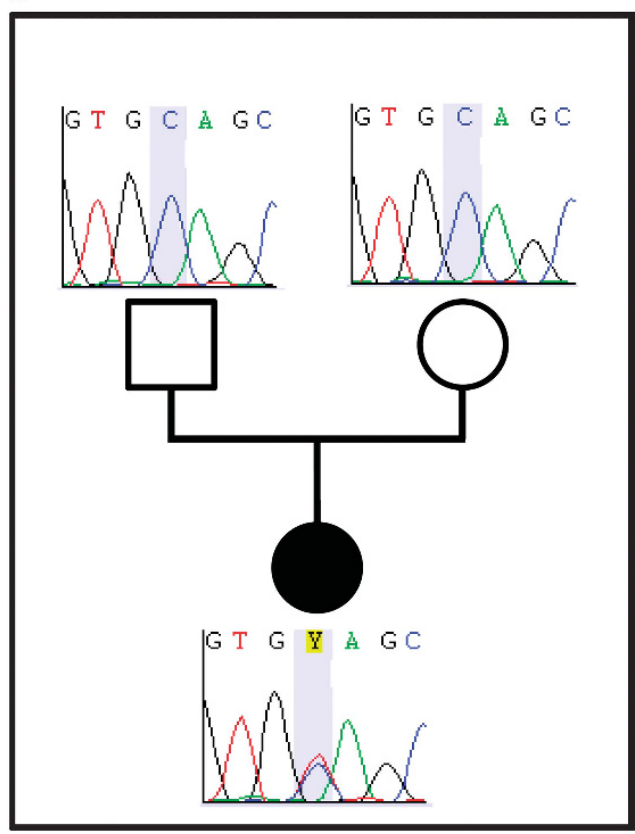

b

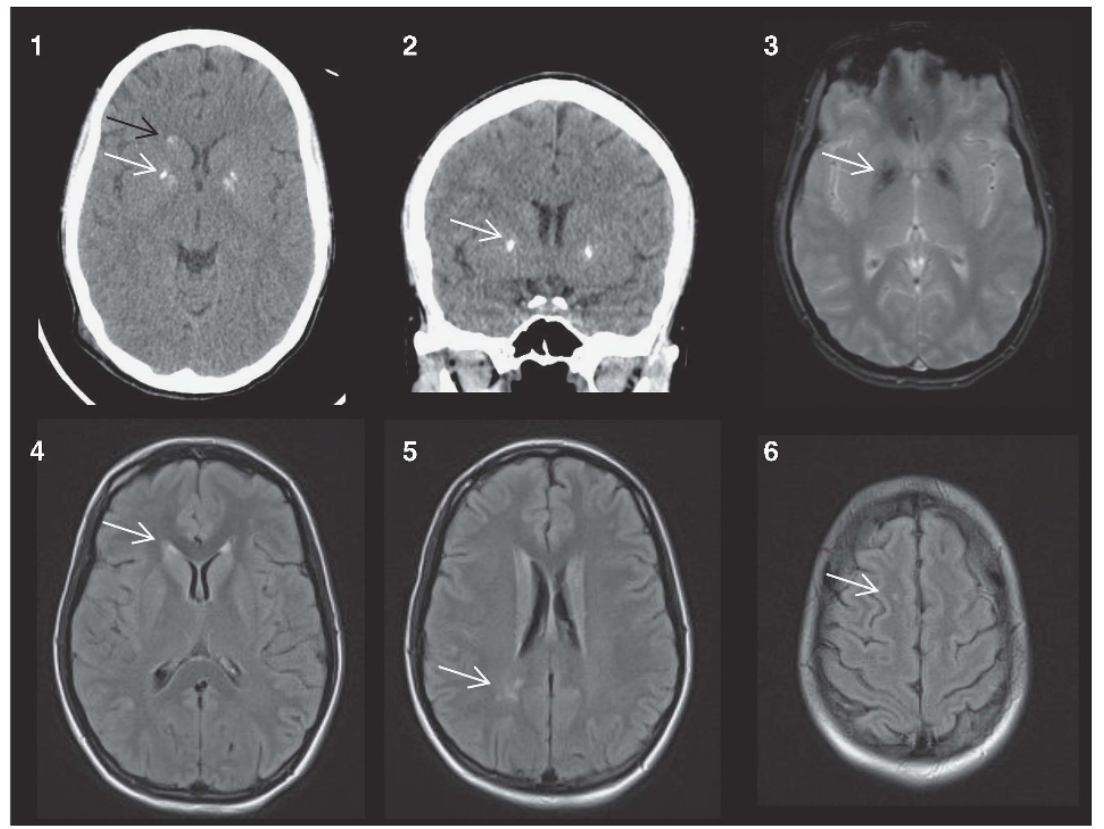

Figure 1 Electropherograms and brain imaging. Electropherograms show a heterozygous substitution of a $\mathrm{C}$ by a $\mathrm{T}$ in the proband, and a wild-type allele (C) in both parents (a). Brain CT scans on axial (b-1) and coronal (b-2) views show moderate calcifications in the lenticular nuclei (white arrows) and faint calcifications in the caudate nuclei (black arrow). On T2*-weighted MRI, calcifications appear hypointense (b-3) while no signal modification is seen in the lenticular nuclei in FLAIR-weighted images, showing hyperintensities on anterior periventricular regions (b-4) and subcortical white matter (b-5, b-6). 
We demonstrate here that a true sporadic occurrence of IBGC, due to a causative de novo mutation, can occur.

The mutation occurred at position c.439 (p.147), just between two recently reported nonsense mutations in $P D G F B$ (resulting in p.Gln145* and p.Arg149*). Interestingly, the c.445C > T (p.Arg149*) mutation also most likely occurred de novo, although, to our knowledge, paternity was not verified..$^{5}$ This suggests a clustering of de novo mutations in exon 4 of the PDGFB gene. Such a phenomenon has been studied using pangenomic data from concordant monozygotic twins with autism, and a mutability index (MI) was calculated for each exon as an estimate of relative mutation rate at single-nucleotide resolution. ${ }^{10}$ The mean MI is 2.41 for exon 4 of PDGFB (chr22:37,957,573_37,957,779, hg18), the genome-wide arithmetic mean of MI being 1, and the mean MI of all other exons of PDGFB being less than 1.54, except for exon 5 (2.15). In accordance with these estimates, our data suggest that exon 4 of PDGFB could be a hotspot for single-nucleotide substitutions. It is also well-recognized that an older paternal age is an extrinsic factor favoring de novo point mutations. ${ }^{10}$ Although the parental origin of the mutated allele could not be documented in our case, we note that the father was aged 44 and the mother 29 at their daughter's date of birth.

Surprisingly, we did not find further mutations of $P D G F B$ with the exception of the previously reported one, ${ }^{5}$ among the familial cases and those with sporadic presentation of our case series. De novo occurrence of a $P D G F B$ mutation is therefore probably a quite rare event among the IBGC cases. Nevertheless, among the seven mutations reported within $P D G F B$ so far, two occurred de novo, which is a relevant result.

This c.439C $>\mathrm{T}\left(\mathrm{p} . \mathrm{Gln} 147^{\star}\right)$ mutation might not be subject to efficient NMD because it is located close to the exon4-exon5 junction, ${ }^{11}$ which could explain that we did not find mRNA decay. However, the mutation is clearly detrimental, as it is predicted to result in a shortened protein with loss of functionally critical domains including interface-contributing residues of the PDGF-B:PDGFR $\beta$ complex. ${ }^{12}$
To conclude, we demonstrate true sporadic occurrence of IBGC due to a de novo nonsense mutation. Our results further support the involvement of the loss of function of PDGF-B in IBGC and suggest a possible hotspot for single-nucleotide substitutions in exon 4 of PDGFB.

\section{CONFLICT OF INTEREST}

The authors declare no conflict of interest.

\section{ACKNOWLEDGEMENTS}

We thank Tracey Avequin for her help in editing the manuscript.

1 Nicolas G, Pottier C, Charbonnier C et al: Phenotypic spectrum of probable and genetically-confirmed idiopathic basal ganglia calcification. Brain 2013; 136 3395-3407.

2 Wang C, Li Y, Shi L et al: Mutations in SLC20A2 link familial idiopathic basal ganglia calcification with phosphate homeostasis. Nat Genet 2012; 44: 254-256.

3 Hsu SC, Sears RL, Lemos RR et al: Mutations in SLC20A2 are a major cause of familial idiopathic basal ganglia calcification. Neurogenetics 2013; 14: 11-22.

4 Nicolas G, Pottier C, Maltete D et al: Mutation of the PDGFRB gene as a cause of idiopathic basal ganglia calcification. Neurology 2013; 80: 181-187.

5 Keller A, Westenberger A, Sobrido MJ et al: Mutations in the gene encoding PDGF-B cause brain calcifications in humans and mice. Nat Genet 2013; 45: 1077-1082.

6 de Oliveira MF, Steinberg SS, de Oliveira JR: The challenging interpretation of genetic and neuroimaging features in basal ganglia calcification. Gen Hosp Psychiatry 2013; 35: 210-211.

7 Smits MG, Gabreels FJ, Froeling PG, de Abreu RA, Thijssen HO, Renier WO Calcium-phosphate metabolism in autosomal recessive idiopathic strio-pallido-dentate calcinosis and Cockayne's syndrome. Clin Neurol Neurosurg 1983; 85: 145-153.

8 Colosimo C, Suppa A, Fabbrini G, Bologna M, Berardelli A: Craniocervical dystonia: clinical and pathophysiological features. Eur J Neurol 2010; 17(Suppl 1): 15-21.

9 Wszolek ZK, Baba Y, Mackenzie IR et al: Autosomal dominant dystonia-plus with cerebral calcifications. Neurology 2006; 67: 620-625.

10 Michaelson JJ, Shi Y, Gujral M et al: Whole-genome sequencing in autism identifies hot spots for de novo germline mutation. Cell 2012; 151: 1431-1442.

11 Schweingruber C, Rufener SC, Zund D, Yamashita A, Muhlemann O: Nonsensemediated mRNA decay-mechanisms of substrate mRNA recognition and degradation in mammalian cells. Biochim Biophys Acta 2013; 1829: 612-623.

12 Shim AH, Liu H, Focia PJ, Chen X, Lin PC, He X: Structures of a platelet-derived growth factor/propeptide complex and a platelet-derived growth factor/receptor complex. Proc Natl Acad Sci USA 2010; 107: 11307-11312.

Supplementary Information accompanies this paper on European Journal of Human Genetics website (http://www.nature.com/ejhg) 